\title{
LIGKA: A Linear Gyrokinetic Code for the Description of Background Kinetic and Fast Particle Effects on the MHD Stability in Tokamaks
}

\author{
Ph. Lauber*, S. Günter*, A. Könies ${ }^{+}$, S.D. Pinches* \\ *Max-Planck-Institut für Plasmaphysik, EURATOM-Association, Boltzmannstr. 2, \\ D-85748 Garching \\ ${ }^{+}$Max-Planck-Institut für Plasmaphysik, Teilinstitut Greifswald, \\ EURATOM-Association, Wendelsteinstr. 1, D-17491 Greifswald
}

\begin{abstract}
In a plasma with a population of super-thermal particles generated by heating or fusion processes, kinetic effects can lead to the additional destabilisation of MHD modes or even to additional energetic particle modes. In order to describe these modes, a new linear gyrokinetic MHD code has been developed and tested, LIGKA (Linear Gyrokinetic Shear Alfvén Physics) [1; 2], based on a gyrokinetic model [3]. A finite Larmor radius expansion together with the construction of some fluid moments and specification to the shear Alfvén regime results in a self-consistent, electromagnetic, non-perturbative model, that allows not only for growing or damped eigenvalues but also for a change in mode-structure of the magnetic perturbation due to the energetic particles and background kinetic effects.

Compared to previous implementations [3], this model is coded in a more general and comprehensive way. LIGKA uses a Fourier decomposition in the poloidal coordinate and a finite element discretisation in the radial direction. Both analytical and numerical equilibria can be treated. Integration over the unperturbed particle orbits is performed with the drift-kinetic HAGIS code $[23 ; 24]$ which accurately describes the particles' trajectories. This allows finite-banana-width effects to be implemented in a rigorous way since the linear formulation of the model allows the exchange of the unperturbed orbit integration and the discretisation of the perturbed potentials in the radial direction.

Successful benchmarks for toroidal Alfvén eigenmodes (TAEs) and kinetic Alfvén waves (KAWs) with analytical results, ideal MHD codes, drift kinetic codes and other codes based on kinetic models are reported.
\end{abstract}

Key words: Gyrokinetics, MHD, Fast Particle Effects PACS: 52.65.Tt, 52.55.Pi 


\section{Introduction}

The closer magnetic fusion experiments approach ignition the more interest and concern is attracted by super-thermal particles and their effect on stability and transport. Especially large scale MHD instabilities are among the most dangerous modes for future fusion devices. Due to their global structure they can efficiently tap energy from the free gradients of the fast particle distribution, grow to large amplitudes, expell hot particles and thus decrease the heating efficiency and cause damage to the first wall.

These modes usually have frequencies in the range of the plasma's Alfvén frequency (for typical parameters $\sim 10^{6} / s$ ) i.e. well below the ion cyclotron frequency $\left(\sim 10^{8} / s\right)$. A large scale structure means that these perturbations have low $m$ (poloidal) and low $n$ (toroidal) mode numbers. Therefore they cannot be described locally or in the ballooning (i.e. high-n) approximation [7].

When the properties of an ignited plasma are predicted, especially toroidal Alfvén eigenmodes modes (TAEs) attract increasing interest: TAEs can be excited in 'gaps' that are caused by the break up of the continuous Alfvén spectrum due to toroidal coupling. In such a gap, there is no continuum damping present. This allows the existence of global modes [13; 14] that can be driven to large amplitudes by passing or trapped energetic ions [16] with dangerous consequences to confinement and stability.

The traditional description with ideal MHD codes cannot take into account kinetic effects such as wave-particle interaction, finite-Larmor-radius (FLR) and parallel electric field effects which are crucial to determine the mode stability $[11 ; 17]$. In order to include these kinetic effects, hybrid models were used extensively: for hot particles, the gradient $\nabla P$ term is replaced by $\nabla\left(P+P_{h o t}\right)$ derived from the kinetic equations. This leads to an extension of the energy principle and this extended system is then solved in a perturbative way, i.e. only the eigenvalue and not the eigenfunction is allowed to change. The most important numerical codes based on this model are CASTOR-K [18] and NOVA-K [15], the extensions of the resistive MHD spectral stability codes CASTOR [26] resp. NOVA. Furthermore, a complex resisity model has been employed [20] to descibe background kinetic effects with CASTOR-K.

This perturbative treatment is only valid for small fast particle pressures and for cases where there is no coupling to small-scale modes. Therefore nonperturbative models are required to explain discrepancies that have been found between experimental data and the predicitons of perturbative codes. Also modes that only exist in the presence of fast particles, energetic particle modes (EPMs), demand this non-perturbative treatment.

On the non-perturbative side, there is the dielectric tensor model [27] and its numerical implementation PENN [21]. With this code it has been shown that the non-perturbative treatment indeed becomes crucial for cases where e.g. radiative damping via coupling the the kinetic Alfvén wave (KAW) $[11 ; 17 ; 25]$ 
is important. However, some of the results have not been confirmed by other codes or by analytical estimates.

LIGKA is based on a linear, self-consistent, non-perturbative, gyrokinetic model [3]-[6] which will be described in section 2. Compared to the derivation and numerical implementation (KIN2DEM) described in references [3]-[6], the equations here are kept more general: some of the analytical expansions as used in KIN2DEM are replaced by more accurate numerical evaluations. Also the gyro-operators in the GKM were kept for consitency.

Section 3 gives a detailed desciption of the numerical implementation, i.e. the discretisation of the operators, the evaluation of the propagator integrals, the Landau-resonance-integral problem and the overall strategy for solving this non-linear eigenvalue problem.

Section 4 shows a series of benchmarks with analytical results, ideal-MHD codes, the drift-kinetic CAS3D-K code and the KIN2DEM code [3].

Finally, conclusions and an outlook to future applications and improvements are given.

\section{Model}

Based on a linear gyrokinetic formulation, a model consisting of the following three equations was previously developed [3]-[6]: the quasi-neutrality equation $(\mathrm{QN})$

$$
\sum_{a} \frac{e_{a}^{2} n_{a}}{T_{a}}\left[\varrho_{a}^{2} \nabla_{\perp}^{2}\right] \phi+e_{a} \int J_{0} f_{a} d^{2} \mathbf{v}=0 ; \quad \mathbf{E}=-\nabla \phi-\frac{\partial \mathbf{A}}{\partial t}
$$

the gyrokinetic moment equation (GKM)

$$
\begin{aligned}
& -\omega^{2}\left[\nabla \cdot\left(\frac{1}{v_{A}^{2}} \nabla_{\perp} \phi\right)\right]+(\mathbf{B} \cdot \nabla) \frac{\left(\nabla \times \nabla \times(\nabla \psi)_{\|}\right) \cdot \mathbf{B}}{B^{2}}+\left[\nabla(\nabla \psi)_{\|} \times \mathbf{b}\right] \cdot \nabla \frac{\mu_{0} j_{0 \|}}{B}= \\
& -\sum_{a} i \omega \mu_{0} \int d^{2} v\left(e \mathbf{v}_{d} \cdot \nabla J_{0} f\right)_{a}+\omega^{2} \frac{3}{4} \frac{\mu_{0} e_{a}^{2} n_{a}}{T_{a}} \varrho_{a}^{4} \nabla_{\perp}^{4} \phi
\end{aligned}
$$

and the gyrokinetic equation (GKE) itself:

$$
\begin{aligned}
& \frac{\partial f_{a}}{\partial t}+\left(U_{a} \mathbf{b}+\mathbf{v}_{d, a}\right) \cdot \nabla f_{a}= \\
& \frac{\mathbf{b}}{e_{a} B} \cdot\left(\nabla F_{0, a} \times \nabla H_{1, a}\right)+\frac{\partial F_{0, a}}{\partial E}\left(U_{a} \mathbf{b}+\mathbf{v}_{d, a}\right) \cdot \nabla H_{1, a}
\end{aligned}
$$


In these equations, $\sum_{a}$ stands for the summation over the particle species (background ions and electrons, fast ion population), $e_{a}$ for the particle charge, $n_{a}$ and $T_{a}$ for density and temperature, $\varrho_{a}$ for the gyro radius, $J_{0}=J_{0}\left(k_{\perp} \varrho_{a}\right)$ for the 0 -th order Bessel function originating from the gyro-angle average

$$
\frac{1}{2 \pi} \int d \xi e^{ \pm \varrho \cdot \nabla}=\frac{1}{2 \pi} \int d \xi e^{ \pm \varrho \nabla_{\perp} \cos \xi}=J_{0}\left(\frac{\varrho \nabla_{\perp}}{i}\right)
$$

$\int d^{2} \mathbf{v}$ for the integration over the two remaining variables in velocity space, $v_{A}$ for the Alfvén velocity, $j_{0 \|}$ for the parallel equilibrium current, $U$ for the velocity parallel to the equilibrium magnetic field $\mathbf{B}, v_{d}$ for the drift velocity of the particles, $F_{0}$ for the equilibrium particle distribution function and $H_{1}=e_{a} J_{0}\left(\phi-\frac{k_{\|} U}{\omega} \psi\right)$ for the perturbed Hamiltonian.

The unknown variables are the electrostatic potential $\phi$, the potential $\psi$ for the parallel component of the electromagnetic potential $A_{\|}=\frac{1}{i \omega}(\nabla \psi)_{\|}$and the perturbed distribution function $f$. Note, that the eigenfrequency $\omega$ appears non-linearly within the propagator and velocity space integrals.

The equations above were derived by setting $\mathbf{A}_{\perp}=\mathbf{0}$. This simplification together with a low- $\beta$ assumption implies that the modes under consideration are almost incompressible shear Alfvén modes with small parallel magnetic perturbations and small pressure perturbations. For arbitrary $\beta$ one would have to solve one additional equation for the perturbed parallel magnetic field $\delta B_{\|}$as derived in the framework of kinetic ballooning theory [8]. However, if $\beta$ is assumed to be small but nevertheless the coupling to the sound waves has to be kept, a set of two slightly modified equations can be derived which will be reported elsewhere. The present version of LIGKA neglects the coupling to the sound wave which is a good approximation for the modes under consideration in this paper.

The GKE is further transformed by substituting [9]:

$$
f_{a}=h_{a}+H_{1, a} \frac{\partial F_{0, a}}{\partial E}-\left[e_{a} \frac{\partial F_{0, a}}{\partial E}-\frac{\nabla F_{0, a}}{i \omega B} \cdot(\mathbf{b} \times \nabla)\right] J_{0} \psi
$$

This substitution creates terms proportional to $\phi-\psi$ that contain the $E_{\|^{-}}$ effects. Close to the MHD-limit, they become small compared to the $\omega_{*}$-term. As shown below, this $\omega_{*}$-term gives the lowest order solution of the GKE, leading to an MHD-like pressure term. Expressions proportional to $U$ vanish during integration over velocity space, if an equilibrium distribution symmetric in $U$ is chosen. This is true for background species (no rotation assumed) and typical $\alpha$-particle distributions. Equations that take into account also asymmetry in U (e.g. caused by NBI heating) have been derived and will be published elsewhere.

Substituting $h$ in favour of $f$ in the QN and GKM equations, assuming a Maxwellian equilibrium distribution function $F_{0}$ and integrating over velocity space results for the QN equation in (the index $a$ is omitted for simplicity): 


$$
e \int J_{0} h d^{3} \mathbf{v}+\frac{e^{2} n_{0}}{T} \Gamma_{0}\left[\psi-\phi-\left(1+\eta G_{0}\right) \frac{\omega_{*}}{\omega} \psi\right]=-\frac{e^{2} n_{0}}{T}\left[\varrho^{2} \nabla_{\perp}^{2}\right] \phi
$$

where

and

$$
\omega_{*} \equiv\left[\frac{T \mathbf{b}}{i e B} \times \frac{\nabla n_{0}}{n_{0}} \cdot \nabla\right] ; \quad \eta \equiv \frac{\nabla T}{T} / \frac{\nabla n_{0}}{n_{0}}
$$

$$
\chi \equiv \frac{v_{t h}^{2} k_{\perp}^{2}}{\Omega^{2}} ; \quad \Gamma_{0}(\chi)=e^{-\chi} I_{0}(\chi) ; \quad 2 G_{0}(\chi)=-\chi+\chi I_{1}(\chi) / I_{0}(\chi),
$$

$v_{t h}^{2}=T / m, \Omega^{2}=e B / m$ and $I_{n}$ as the modified Bessel functions of $n$-th order. Using

$$
\omega_{d} \equiv \frac{\mathbf{v}_{d} \cdot \nabla}{i} \quad \text { and } \quad \mathbf{v}_{d}=\frac{1}{m \Omega B}\left(\mu B+m U_{\|}^{2}\right)(\mathbf{b} \times \nabla B)
$$

which is true for low- $\beta$, and partially carrying out the integration over velocity space, the first term on the right hand side of the GKM becomes:

$$
\begin{aligned}
& \omega^{2} e \mu_{0} \int d^{2} v \frac{\omega_{d}}{\omega} J_{0} f=\mu_{0} e \omega^{2} \int d^{2} v \frac{\omega_{d}}{\omega} J_{0} h+\frac{2 \mu_{0} e \omega^{2}}{B^{2}}(\mathbf{b} \times \nabla B) \cdot \nabla \\
& \left\{n_{0} \Gamma_{0}(\chi)\left[\psi-\phi-\left[(1+\eta)\left(1+G_{0}(\chi)+\eta G_{1}(\chi)\right] \frac{\omega_{*}}{\omega}\right] \psi\right\}\right.
\end{aligned}
$$

with $G_{1}(\chi)=\chi\left(I_{1} / I_{0}-3 / 2\right)-\chi^{2}\left(I_{1} / I_{0}-1\right)$. Following reference [3], contact to ideal MHD can be established by leaving out $E_{\|}$-effects (setting $h$ and $\phi-\psi$ zero), going into the zero gyroradius limit $(\chi=0)$ and applying

$$
-\sum_{i, e} e n_{0}[1+\eta] \frac{\omega_{*}}{\omega}=\sum_{i, e}\left[\frac{T \nabla n_{0}+n_{0} \nabla T}{i \omega B} \times \mathbf{b}\right] \cdot \nabla=\frac{\nabla[P(r)]}{i \omega B} \cdot(\mathbf{b} \times \nabla) .
$$

Then the GKM reads:

$$
\begin{aligned}
& -\omega^{2}\left[\nabla \cdot\left(\frac{1}{v_{A}^{2}} \nabla_{\perp} \phi\right)\right]+(\mathbf{B} \cdot \nabla) \frac{\left(\nabla \times \nabla \times(\nabla \psi)_{\|}\right) \cdot \mathbf{B}}{B^{2}} \\
& +\left[\nabla(\nabla \psi)_{\|} \times \mathbf{b}\right] \cdot \nabla \frac{\mu_{0} j_{0 \|}}{B}+2 \mu_{0}\left[\frac{\mathbf{b}}{B} \times \frac{\nabla B}{B}\right] \cdot \nabla\left[\frac{\nabla P}{B}(\mathbf{b} \times \nabla) \psi\right]=0 .
\end{aligned}
$$

This equation can also be derived starting from the ideal MHD equations [3]. The QN is trivially satisfied in this limit. When FLR-effects are kept, another limit called the 'reduced kinetic model' can be derived [2]. These two limits will be recovered numerically in the validation and benchmark section of this paper.

Now the expressions proportional to $h$ are further manipulated: using the ansatz

$$
h_{a}(t, \mathbf{x})=\bar{h}_{a}(r, \theta) e^{i(n \varphi-\omega t)}
$$


where $\varphi$ and $n$ are the toroidal angle and mode number, the GKE becomes:

$$
\begin{aligned}
& \bar{h}_{a}=i e_{a} \sum_{m} \int_{-\infty}^{t} d t^{\prime} e^{i\left[n\left(\varphi^{\prime}-\varphi\right)-m\left(\theta^{\prime}-\theta\right)-\omega\left(t^{\prime}-t\right)\right]} e^{-i m \theta} . \\
& \frac{\partial F_{0, a}}{\partial E}\left(\omega-\omega_{*}^{T}\right) J_{0} \cdot\left[\phi_{m}\left(r^{\prime}\right)-\left(1-\frac{\omega_{d}\left(\theta^{\prime}\right)}{\omega}\right) \psi_{m}\left(r^{\prime}\right)\right]
\end{aligned}
$$

Here, a Fourier expansion in the poloidal angle $\theta$ with the poloidal mode number $m$ has been performed. The time integral in equation (8) has to be carried out along the unperturbed particle orbit, indicated by the primed quantities $t^{\prime}, r^{\prime}, \theta^{\prime}$ and $\varphi^{\prime}$.

\subsection{Circulating Particles}

After expanding into bounce harmonics labelled by the index $k$, changing to the the velocity space coordinates $Y=E / T$ and $\Lambda=\mu B_{0} / E$, considering only first order terms for the drifts, integrating over time and applying the projection operator $\int_{-\pi}^{\pi} \frac{d \theta}{2 \pi} e^{i p \theta}$, the circulating particle contribution becomes:

$$
\begin{aligned}
& \left(\int_{-\pi}^{\pi} \frac{d \theta}{2 \pi} e^{i p \theta} \int J_{0} h d^{3} \mathbf{v}\right)^{c i r c}=-\pi e_{a} v_{t h}^{3} \sum_{m} \int_{0}^{b_{\min }(r)} d \Lambda \int_{0}^{\infty} d Y \sqrt{Y} \\
& \sum_{k} \frac{\partial F_{0}}{\partial E} \frac{\left(\omega-\hat{\omega}_{*}\right) \omega}{\omega^{2}-\left(S_{m}+k\right)^{2} \omega_{t}^{2}} K_{m, p, k} \cdot J_{0}^{2}\left[\hat{a}_{k m} \phi_{m}(r)-\left(\hat{a}_{k m}-\frac{\hat{a}_{k m}^{G} \bar{\omega}_{d}(r)}{\omega}\right) \psi_{m}(r)\right]
\end{aligned}
$$

with

$$
\begin{aligned}
& K_{m, p, k}=\frac{1}{2 \pi} \int_{-\pi}^{\pi} \frac{d \theta}{b(r, \theta) \sqrt{1-\frac{\Lambda}{b(r, \theta)}}} e^{-i\left[S_{p} \theta-\left(k+S_{m}\right) \omega_{t} \hat{t}(\theta)\right]}, \\
& \hat{a}_{m, k}=\frac{1}{\tau_{t}} \int_{-\tau_{t} / 2}^{\tau_{t} / 2} d \hat{t}^{\prime} e^{i\left[S_{m} \theta^{\prime}-\left(k+S_{m}\right) \omega_{t} \hat{t}^{\prime}\right]} ; \quad \hat{a}_{m, k}^{G}=\frac{1}{\tau_{t}} \int_{-\tau_{t} / 2}^{\tau_{t} / 2} d \hat{t}^{\prime} \frac{\omega_{d}\left(r^{\prime}, \theta^{\prime}\right)}{\bar{\omega}_{d}(r)} e^{i\left[S_{m} \theta^{\prime}-\left(k+S_{m}\right) \omega_{t} \hat{t}^{\prime}\right]}
\end{aligned}
$$

and

$$
S_{p}=n q(r)-p ; \quad S_{m}=n q(r)-m .
$$

For circulating particles the integration over $\Lambda$ has to be carried out from 0 to $b_{\min }(r)=\min [b(r, \theta)]=\min \left[B_{0} / B(r, \theta)\right] . \omega_{t}$ and $\tau_{t}$ are the transit frequency 
and time, and

$$
\hat{\omega}_{*}=\frac{\nabla F_{0} \times \mathbf{b}}{i e B \frac{\partial F_{0}}{\partial E}} \cdot \nabla
$$

Also the sum over co- and counter-passing particles has been performed already assuming symmetry in velocity space.

The operator $J_{0}^{2}\left[k_{\perp}(r, \theta) \varrho\right]$ can be treated in three different ways:

- If the mode structure permits, it's radial derivatives are completely neglected and only the $\theta$ - dependent terms are taken into account. This is how e.g. KIN2DEM is set up. $k_{\perp}$ simply reduces to $k_{y}=-m / r$.

- A more accurate way is to use an intermediate result for the eigenfunction: since the eigenvalue is calculated iteratively, a guess for $\phi$ and $\psi$ during run-time is available. As initial guess the pure MHD eigenfunction or an eigenfunction calculated with the approximation method given above can be employed. Also results from the 'antenna'-version of LIGKA [2] can be used.

This method was tested so far only for cases where the eigenfunctions did not have any zero crossings: $\phi^{\prime \prime} / \phi$ and $\phi^{\prime} / \phi$ were therefore always a well behaved functions without singularities.

- The operator can be expanded up to second order and the result added to the contributions of the first and second order derivative coefficients from the MHD part. This treatment will be tested in a future version of LIGKA.

\subsection{Trapped Particles}

For trapped particles the drift effects due to the drift operator on the left hand side of the GKE (equation 3) are taken into account: in a linear description, where the orbit integrals are performed over unperturbed orbits it is possible to change the order of time integration and the discretisation of the perturbed potentials in radial direction. Thus, the time integral can be written as a sum of integrals:

$$
\int_{-\infty}^{t} d t^{\prime}=\int_{t_{1}}^{t_{2}} d t^{\prime}+\int_{t_{2}}^{t_{3}} d t^{\prime}+\ldots=\sum_{j^{\prime}} \int_{t_{j^{\prime}}}^{t_{j^{\prime}+1}} d t^{\prime}
$$

The index $j^{\prime}$ counts the finite element partitions in the radial coordinate: the particle spends the time $t_{j^{\prime}+1}-t_{j^{\prime}}$ in the radial bin with number $j^{\prime}$ (see figure $1)$.

Before proceeding, the phase factor

$$
e^{i\left[n\left(\varphi^{\prime}-\varphi\right)-m\left(\theta^{\prime}-\theta\right)-\omega\left(t^{\prime}-t\right)\right]}
$$




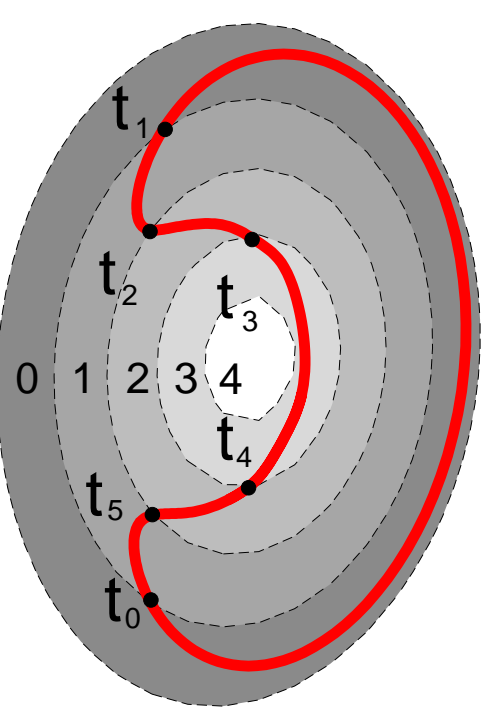

Fig. 1. The orbit for trapped particles is split up in pieces corresponding to the finite element discretisation

is rewritten in a more convenient way using the following definitions [10]:

$$
\begin{aligned}
& n\left(\varphi^{\prime}-\varphi\right)-m\left(\theta^{\prime}-\theta\right)=\int_{t}^{t^{\prime}} d t^{\prime \prime}\left(n \frac{d \varphi}{d t^{\prime \prime}}-m \frac{d \theta}{d t^{\prime \prime}}\right) \\
& \omega_{D}=n\left[\frac{d \varphi}{d t}-q\left(r^{0}\right) \frac{d \theta}{d t}\right] ; \quad \omega_{D}^{0}=\frac{1}{\tau_{b, t}} \int d t \omega_{D}
\end{aligned}
$$

$r^{0}$ is the orbit averaged radial position of a particle.

$$
W=W(t)=\int_{0}^{t} d t^{\prime \prime} \Delta \omega_{D} ; \quad W^{\prime}=W\left(t^{\prime}\right)=\int_{0}^{t^{\prime}} d t^{\prime \prime} \Delta \omega_{D} ; \quad \Delta \omega_{D}=\omega_{D}-\omega_{D}^{0}
$$

The time integration has to be started at $t^{\prime} \rightarrow-\infty$. Taking into consideration that the orbit motion is periodic, one obtains:

$$
\begin{aligned}
& \int_{-\infty}^{t} d t^{\prime} e^{-i\left(\omega-\omega_{D}^{0}-k \omega_{b}\right)\left(t^{\prime}-t\right)} \psi\left(r^{\prime}\right)= \\
= & \sum_{\kappa=0}^{\infty} \int_{t-(\kappa+1) \tau_{b}}^{t-\kappa \tau_{b}} d t^{\prime} e^{-i\left(\omega-\omega_{D}^{0}-k \omega_{b}\right)\left(t^{\prime}-t\right)} \psi\left(r^{\prime}\right)= \\
= & \sum_{\kappa=0}^{\infty} \sum_{j=0}^{N-1} \int_{t-\kappa \tau_{b}-t_{j+1}}^{t-t_{j}} d t^{\prime} e^{-i\left(\omega-\omega_{D}^{0}-k \omega_{b}\right)\left(t^{\prime}-t\right)} \psi\left(r_{j}\right)= \\
= & \sum_{\kappa=0}^{\infty} \sum_{j=0}^{N-1} \frac{\left[e^{-i\left(\omega-\omega_{D}^{0}-k \omega_{b}\right)\left(-\kappa \tau_{b}-t_{j}\right)}-e^{-i\left(\omega-\omega_{D}^{0}-k \omega_{b}\right)\left(-\kappa \tau_{b}-t_{j+1}\right)}\right]}{-i\left(\omega-\omega_{D}^{0}-k \omega_{b}\right)} \psi\left(r_{j}\right)=
\end{aligned}
$$




$$
=\sum_{j=0}^{N-1} \frac{e^{i\left(\omega-\omega_{D}^{0}-k \omega_{b}\right) t_{j}}\left[1-e^{i\left(\omega-\omega_{D}^{0}-k \omega_{b}\right)\left(t_{j+1}-t_{j}\right)}\right]}{-i\left(\omega-\omega_{D}^{0}-k \omega_{b}\right)\left[1-e^{i\left(\omega-\omega_{D}^{0}-k \omega_{b}\right) \tau_{b}}\right]} \psi\left(r_{j}\right)
$$

where the relation

$$
\sum_{\kappa=0}^{\infty} e^{i x \kappa}=\frac{1}{1-e^{i x}}
$$

was used. It can be easily verified that for $N=1, t_{j}=0$ and $t_{j+1}=\tau_{b}$, expression (11) simplifies to the zero-orbit-width result: $-1 / i\left(\omega-\omega_{D}^{0}-k \omega_{b}\right)$. Now we can integrate $h$ over the velocity space and over $\theta$, like above for circulating particles .

Therefore, the final expression for trapped particles is:

$$
\begin{aligned}
& \left(\int_{-\pi}^{\pi} \frac{d \theta}{2 \pi} e^{i p \theta} \int J_{0} h d^{3} \mathbf{v}\right)^{\text {trap }}=-\pi e_{a} v_{t h}^{3} \sum_{m} \sum_{j} \int_{b_{\min }(r)}^{b_{\max }(r)} d \Lambda \int_{0}^{\infty} d Y \sqrt{Y} \frac{\partial F_{0}}{\partial E} \sum_{k} \\
& \frac{\mathcal{R}_{k j} K_{k p}\left(\omega-\hat{\omega}_{*}\right)}{\omega-\omega_{D}^{0}-k \omega_{b}} J_{0}^{2} \cdot\left[\hat{a}_{k m} \phi_{m}\left(r_{j}\right)-\left(\hat{a}_{k m}-\frac{\hat{a}_{k m}^{G} \bar{\omega}_{d}(r)}{\omega}\right) \psi_{m}\left(r_{j}\right)\right]
\end{aligned}
$$

with

$$
\begin{aligned}
\mathcal{R}_{k j} & =\sum_{j=0}^{N-1} \frac{e^{i\left(\omega-\omega_{D}^{0}-k \omega_{b}\right) t_{j}}\left[1-e^{i\left(\omega-\omega_{D}^{0}-k \omega_{b}\right)\left(t_{j+1}-t_{j}\right)}\right]}{1-e^{i\left(\omega-\omega_{D}^{0}-k \omega_{b}\right) \tau_{b}}} \\
K_{k p} & =\frac{1}{2 \pi} \int_{\theta^{-}}^{\theta^{+}} \frac{d \theta}{b(r, \theta) \sqrt{1-\frac{\Lambda}{b(r, \theta)}}} e^{-i\left[(n q(r)-p) \theta+W-k \omega_{b} \hat{t}\right]}
\end{aligned}
$$

It should be noted that contrary to the perturbed potentials $\phi, \psi$ and the quantities in the propagator, the equilibrium quantities $F_{0}, \bar{\omega}_{d}$ and $\hat{\omega}_{*}$ are assumed to stay constant in the radial coordinate along a particle orbit.

Finally, in the GKM equation, an integral of the form

$$
\int i e \frac{\mathbf{v}_{d}}{\omega} \cdot \nabla J_{0} h d^{3} \mathbf{v}
$$

has to be performed.

Furthermore, in the expression for $h$ there is also a term proportional to $\omega_{d}(r) \equiv \mathbf{v}_{d} / i \cdot \nabla:$

$$
\left[\hat{a}_{k m} \phi_{m}\left(r_{j}\right)-\left(\hat{a}_{k m}-\frac{\hat{a}_{k m}^{G} \bar{\omega}_{d}(r)}{\omega}\right) \psi_{m}\left(r_{j}\right)\right]
$$

This drift operator entails radial derivatives of $\psi$ and $\phi$. In principle, these derivatives could be included is the system using a similar procedure as described before for $J_{0}$. But due to the smallness of $\dot{r}$ (one order of $\epsilon=a / R_{0}$ 
smaller) compared to $\dot{\theta}$ and $\dot{\varphi}, \omega_{d}$ is replaced by $\omega_{D}=\dot{\varphi}-q\left(r^{0}\right) \dot{\theta}$. This simplification is not justified, when very low frequencies, close to the sound frequency are investigated since the second order derivatives of $\omega_{d}^{2}$ are needed e.g. for the geodesic acoustic correction of the Alfvén continuum [12]. Equations suited for this limit have been recently derived and will be reported elsewhere. In this work, only high frequency phenomena (compared to the sound frequency) are investigated.

Finally, line (13) can be rewritten as:

$$
\begin{aligned}
& \left(\int_{-\pi}^{\pi} \frac{d \theta}{2 \pi} e^{i p \theta} \int i e_{a} \frac{\mathbf{v}_{d}}{\omega} \cdot \nabla J_{0} h d^{3} \mathbf{v}\right)^{\text {trap }}=-\pi e_{a}^{2} v_{t h}^{3} \sum_{m} \sum_{j} \int_{b_{m i n}(r)}^{b_{\max }(r)} d \Lambda \int_{0}^{\infty} d Y \sqrt{Y} \frac{\partial F_{0}}{\partial E} \sum_{k} \\
& \cdot \mathcal{R}_{k j} \cdot K_{k p}^{G} \cdot \frac{\omega-\hat{\omega}_{*}}{\omega-\omega_{D}^{0}-k \omega_{b}} \cdot\left[\hat{a}_{k m} \phi_{m}\left(r_{j}\right)-\left(\hat{a}_{k m}-\frac{\hat{a}_{k m}^{G} \bar{\omega}_{d}(r)}{\omega}\right) \psi_{m}\left(r_{j}\right)\right] \\
& \left(\int_{-\pi}^{\pi} \frac{d \theta}{2 \pi} e^{i p \theta} \int i e_{a} \frac{\mathbf{v}_{d}}{\omega} \cdot \nabla J_{0} h d^{3} \mathbf{v}\right)^{c i r c}=-\pi e_{a}^{2} v_{t h}^{3} \sum_{m} \int_{0}^{b_{\min }(r)} d \Lambda \int_{0}^{\infty} d Y \sqrt{Y} \frac{\partial F_{0}}{\partial E} \sum_{k} \\
& \cdot K_{m k p}^{G} \cdot \frac{\left(\omega-\hat{\omega}_{*}\right) \omega}{\omega^{2}-\left(S_{m}+k\right)^{2} \omega_{t}^{2}} \cdot\left[\hat{a}_{k m} \phi_{m}(r)-\left(\hat{a}_{k m}-\frac{\hat{a}_{k m}^{G} \bar{\omega}_{d}(r)}{\omega}\right) \psi_{m}(r)\right]
\end{aligned}
$$

As before,

$$
K_{m, p, k}^{G}=\frac{1}{2 \pi} \int_{-\pi}^{\pi} \frac{d \theta}{b(r, \theta) \sqrt{1-\frac{\Lambda}{b(r, \theta)}}} \frac{\omega_{D}\left(r^{\prime}, \theta^{\prime}\right)}{\bar{\omega}_{D}(r)} e^{-i\left[S_{p} \theta-\left(k+S_{m}\right) \omega_{t} \hat{t}(\theta)\right]},
$$

and

$$
K_{k p}^{G}=\frac{1}{2 \pi} \int_{\theta^{-}}^{\theta^{+}} \frac{d \theta}{b(r, \theta) \sqrt{1-\frac{\Lambda}{b(r, \theta)}}} \frac{\omega_{D}\left(r^{\prime}, \theta^{\prime}\right)}{\bar{\omega}_{D}(r)} e^{-i\left[(n q(r)-p) \theta+W-k \omega_{b} \hat{t}\right]}
$$

Now our system of equations is complete consisting of the QN (5) and the GKM $(2,6)$ together with the integrals (10), (12), (14) and (15).

\section{Numerical Implementation}

In this section the structure of the numerical implementation and details of the methods involved are described. 
As sketched in figure 2, we start from an equilibrium given analytically or numerically. At present, there exists an interface for the equilibrium code HELENA [26]. Also HAGIS [23; 24], a Monte-Carlo code in a guiding centre Hamiltonian formulation, usually applied for non-linear wave-particle interaction, can use HELENA equilibria as input. Before one starts to run LIGKA itself one computes the integrals over the unperturbed particle orbits with HAGIS based on a given equilibrium, i.e. one calculates for each $m, p, k, r$, $Y$ and $\Lambda$ the integrals $a_{k m}, K_{m p k}, K_{p k}$ and the corresponding $a_{k m}^{G}, K_{m p k}^{G}, K_{p k}^{G}$. For a typical run with 5 poloidally coupled mode numbers this means approximately one gigabyte of data per species. To be able to choose the points in velocity space in an advantageous way, i.e.more points near the trappedpassing boundary, HAGIS is called iteratively to provide a grid in velocity space on which the required integrals are calculated in a separate step.

For calculating the drift orbit averages that determine the wave-particle resonances, no gyro orbit effects are taken into account. This is a reasonable approximation because firstly the 6-d unperturbed trajectory only differs slightly from the drift kinetic one, at least in an ITER, JET or ASDEX-Upgrade like device. Secondly, for the wave-particle energy transfer it is more crucial to capture numerically the fact that there is a pole rather than it's exact position in the complex plane. Thus, as long as the deviations of 6-d and 5-d trajectories are not too large with respect to background quantities, the resonance integrals in the guiding centre approximation are very accurate. The differential operators are calculated with a General Vector Algebra Package [3] based on MATHEMATICA, allowing for a fast and flexible change between different geometries (cylindrical, straight tokamak, tokamak,circular, shaped).

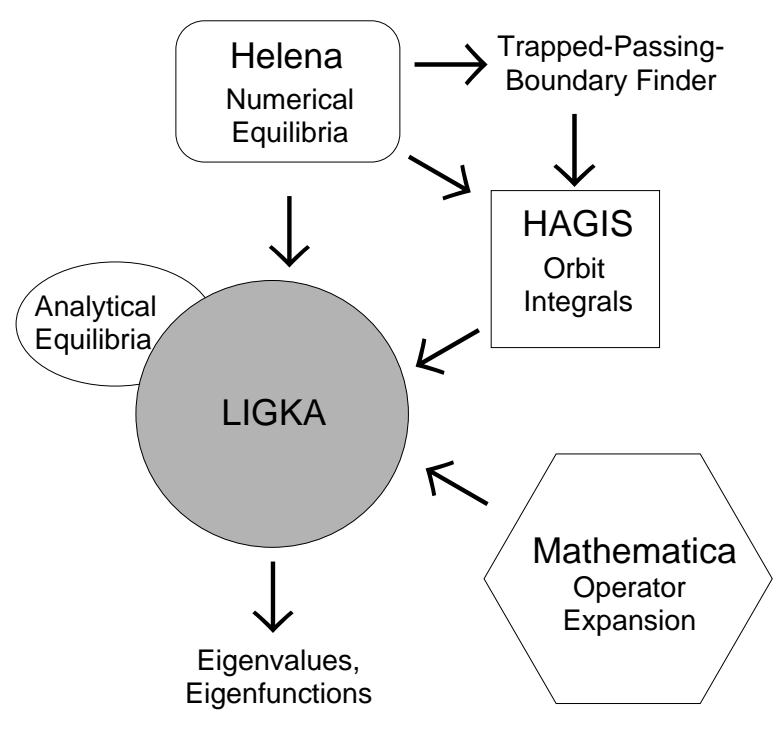

Fig. 2. Survey of different codes involved 


\subsection{Finite Element Discretisation}

As indicated in the last section, LIGKA employs a Fourier decomposition in the toroidal and poloidal angle and finite elements of length $l$ with cubic Hermite polynomials as basis function in the radial coordinate $x$ :

$$
\left\{1-\frac{3 x^{2}}{l^{2}}+\frac{2 x^{3}}{l^{3}}, x-\frac{2 x^{2}}{l}+\frac{x^{3}}{l^{2}}, \frac{3 x^{2}}{l^{2}}-\frac{2 x^{3}}{l^{3}}, \frac{x^{3}}{l^{2}}-\frac{x^{2}}{l}\right\} .
$$

As coordinate system straight field line flux coordinates with $s=\sqrt{\Psi}$ (poloidal flux) are chosen. The toroidal angle is chosen as the geometric angle. The field lines are straight, but not field-aligned. The total matrix is set up using the Galerkin method: the solution is approximated by

$$
\phi_{m}(r)=\sum_{j=1}^{N} \phi_{m j} \varphi_{j}(x), \quad \psi_{m}(r)=\sum_{j=1}^{N} \psi_{m j} \varphi_{j}(x) .
$$

This expansion is substituted into the differential equation and weighted with the basis functions (here given just for $\phi$ ):

$$
\int_{\text {plasma }} d x R(x) \varphi_{k}(x)=0
$$

with

$$
R(x)=A_{p m}\left[\sum_{j=1}^{N} \phi_{m j} \varphi_{j}(x)\right]^{\prime \prime}+B_{p m}\left[\sum_{j=1}^{N} \phi_{m j} \varphi_{j}(x)\right]^{\prime}+C_{p m} \sum_{j=1}^{N} \phi_{m j} \varphi_{j}(x) .
$$

The goal is now to find a set of $\varphi_{k}(x)$ so that the integral above is zero for some choices of the weight functions. By carrying out a partial integration one can transform the differential equations into algebraic ones.

As boundary conditions one imposes that the perturbed potentials for all poloidal harmonics vanish at the the plasma centre (except the the $m=0$ component) and at the outermost flux surface. This is introduced in the system by deleting the first column and the first row as well as the last-but-one column and row for each $m$-block. Finally, a matrix as graphically described in figure 3 is formed.

The basic convergence is checked for an ideal MHD case, where the system is linear in the eigenvalue, and a standard NAG routine can be applied for solving the matrix equation $A \phi=\lambda B \phi$. As shown in figure 4, green line, the relative error of the eigenvalue scales like $l^{-5}$ where $l$ is the length of a finite element and an equidistant grid was chosen. This convergence rate has also been found by other codes based on finite elements [26]. The case shown in figure 4 is an internal kink mode that has a localised structure at the $q=1$ 


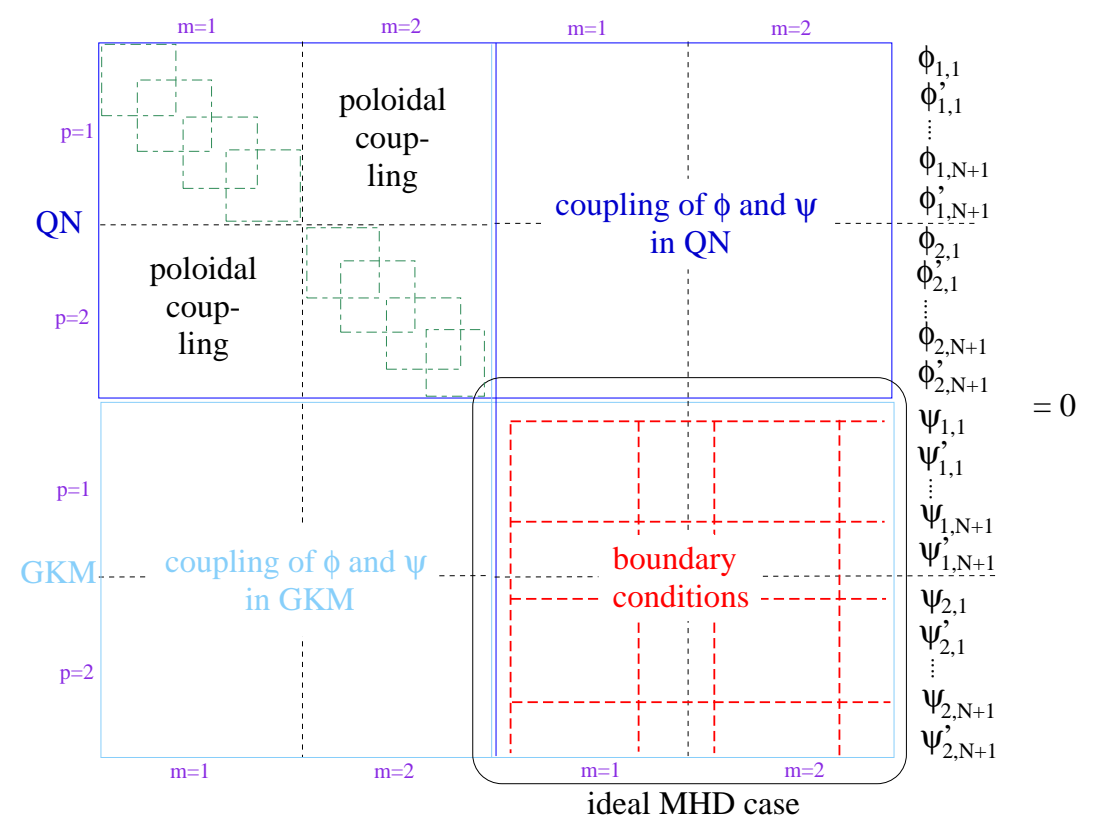

Fig. 3. graphical representation of the complete system for two coupled poloidal modes

surface. This is the reason why with an equidistant grid a $l^{-5}$-convergence can only be found for 100 radial grid points or more (green line in figure 4). LIGKA allows also for mesh accumulation, resulting in a much faster convergence if more points close to the $q=1$ surface are chosen (red dots in figure 4).

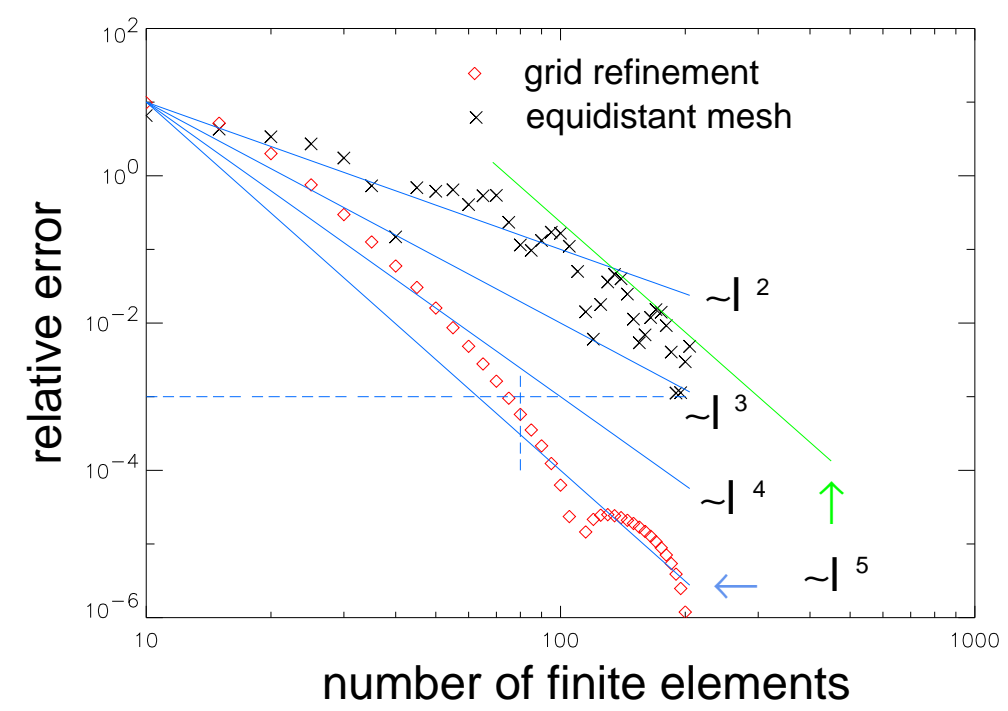

Fig. 4. Relative error of the growth rate of an internal kink mode in dependence of the finite element length $l$ : with an equidistant grid an $l^{-5}$ dependence is only found for more than 100 grid points, with grid refinement around the $q=1$ surface the error scales immediately with $l^{-5}$. 


\subsection{Propagator Integrals}

The bounce and drift frequencies and propagator integrals calculated with HAGIS were benchmarked against analytical formulae, given in [3]. One can see that as expected, for low-energy ions (and of course also electrons, not shown here) the analytical results are recovered accurately (see figure 5). For trapped $\alpha$-particles the analytical expressions become inaccurate and thus deviations can be found.

The propagator integrals involving drifts show the biggest deviations from the analytical formulae: even for low-energy ions in an non-up-down-symmetric elliptical equilibrium, drifts cause substantial differences, even in the sign for energetic particles (figure 6). Thus, for realistic equilibria, the numerical evaluation becomes essential.
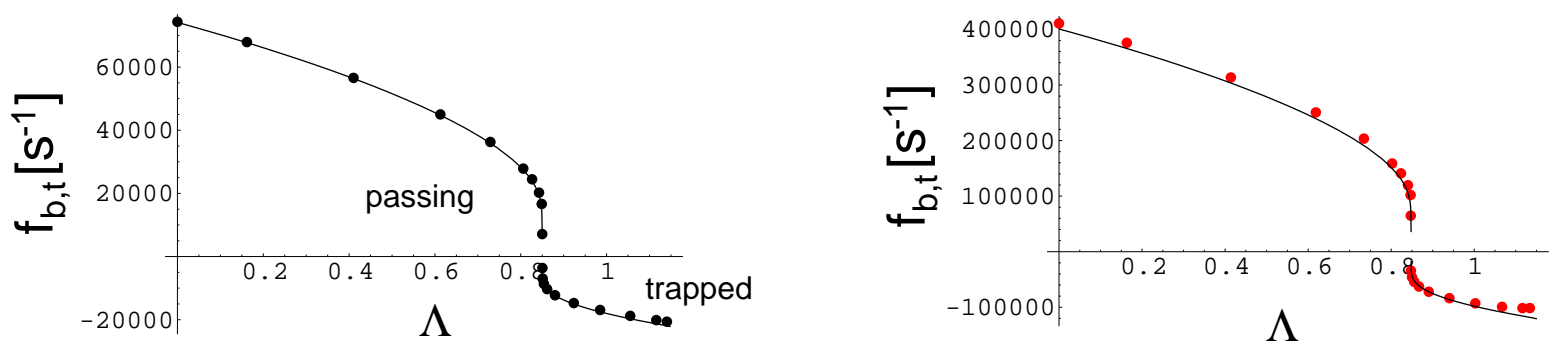

Fig. 5. Circulating/bounce frequency for $28 \mathrm{keV}$ ions (left,dots) and $3.5 \mathrm{MeV}$ alpha particles (right,dots) for a numerical, elliptical equilibrium compared to the analytical formulae (solid line)
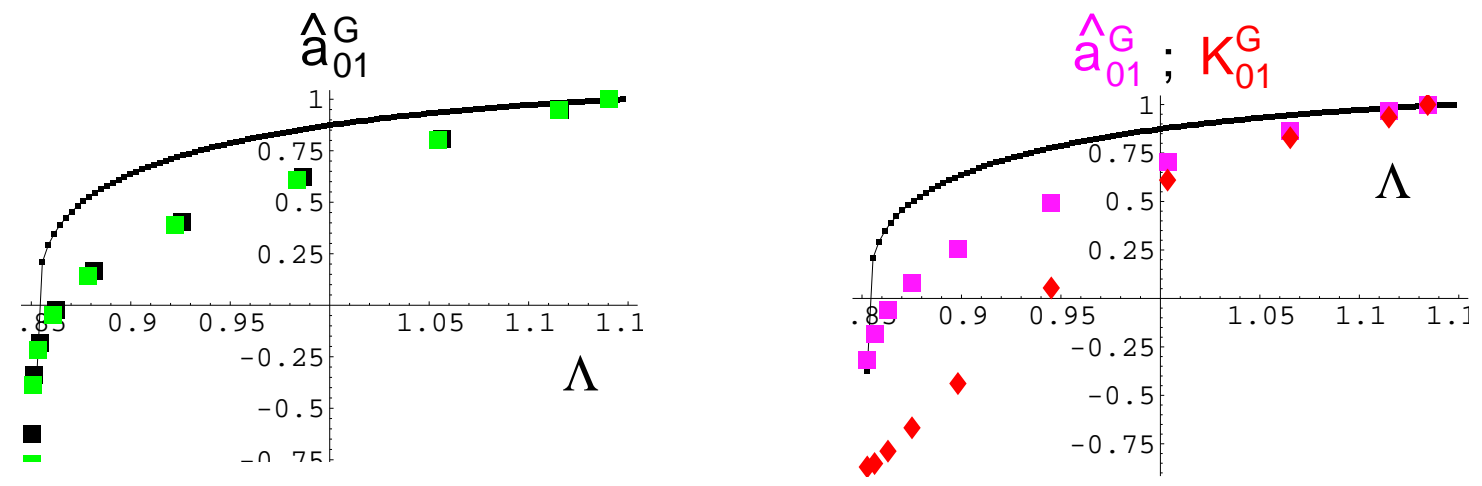

Fig. 6. Left: $\hat{a}_{k m}^{G}$ for $1 \mathrm{keV}$ (black dots) and $140 \mathrm{keV}$ (green dots), $k=0, m=1$, black line: analytical formula) Right: the coefficients $\hat{a}_{k m}^{G}$ (pink diamond) and $K_{k p}^{G}$ ( red dots) for $k=0, m=1,3.5 \mathrm{MeV} \alpha$-particles, elliptic equilibrium 


\subsection{Landau Integrals}

As mentioned before, the kinetic data is given numerically on a $E-\Lambda$-grid. The question now arises how to interpolate or spline this data in order to carry out the resonance integrals $\omega-\omega_{*} / \omega-\omega_{D}-k \omega_{t}$ e.g. in equations 1415 . There are many difficulties connected with this task:

- One typical application of the code is to determine stability boundaries. That means that for these calculations the ratio of damping rate or growth rate $\gamma$ to the real frequency $\omega$ is very small and can be both positive and negative. If now a resonance condition is met the denominator becomes very small and a special Cauchy principal integral algorithm [32] for a near singular function has to be used. Many evaluations of the integrand have to be made.

- Negative imaginary parts of the denominator require the determination of the residual:

$$
\int_{0}^{\Lambda_{\max }} h d \Lambda=P \int_{0}^{\Lambda_{\max }} h d \Lambda+\left.2 \pi i h\left(\Lambda_{z}\right)\left(\Lambda-\Lambda_{z}\right)\right|_{\Lambda \rightarrow \Lambda_{z}}
$$

Here, $\Lambda_{z}$ is the pole in the extended complex $\Lambda$-plane. Numerically, this problem is extremely difficult because either derivatives of the denominator or numerical cancellation of $\Lambda-\Lambda_{z}$ have to be carried out.

- There were predictions about relatively high damping rates for TAE modes at JET [19]. Therefore, any algorithm applied has to be correct also for large imaginary parts. Often the exact position of the pole is not taken into account, but it is rather assumed that the pole lies on the axis.

- The trapped-passing boundary has to be represented accurately because especially barely trapped electrons and fast ions are often in resonance with the modes under investigation.

It was found that rational interpolation solves all the problems mentioned above: applying the Thacher-Tukey algorithm [31], the denominator is written as

$$
\mathcal{D}(\Lambda)=i \gamma+\tilde{\mathcal{D}}(\Lambda)=i \gamma+\frac{a_{0}\left(\Lambda-a_{1}\right)\left(\Lambda-a_{2}\right)}{\left(\Lambda-a_{3}\right)\left(\Lambda-a_{4}\right)}
$$

with real coefficients $a_{0}, \ldots, a_{4}$. In order to include $\gamma$ one solves a simple quadratic equation which yields:

$$
\mathcal{D}(\Lambda)=\frac{c_{0}\left(\Lambda-c_{1}\right)\left(\Lambda-c_{2}\right)}{\left(\Lambda-a_{3}\right)\left(\Lambda-a_{4}\right)}
$$

where $c_{0}, c_{1}$ and $c_{2}$ are now complex. This expression interpolates $\mathcal{D}$ sufficiently, also close to the trapped-passing boundary because it allows for a singularity there (error below $2 \%$, see figure 7 ), is easy and fast to evaluate, 
is trivially continued into complex plane and allows an analytical cancellation of the singularity in the residual term:

$$
\left.2 \pi i \frac{g(z, \Lambda, t)\left(\Lambda-a_{3}\right)\left(\Lambda-a_{4}\right)}{c_{0}\left(\Lambda-c_{2}\right)} \cdot \frac{\left(\Lambda-c_{1}\right)}{\left(\Lambda-c_{1}\right)}\right|_{\Lambda \rightarrow c_{1}}
$$

The singularity $c_{2}$ lies out of the integration range.
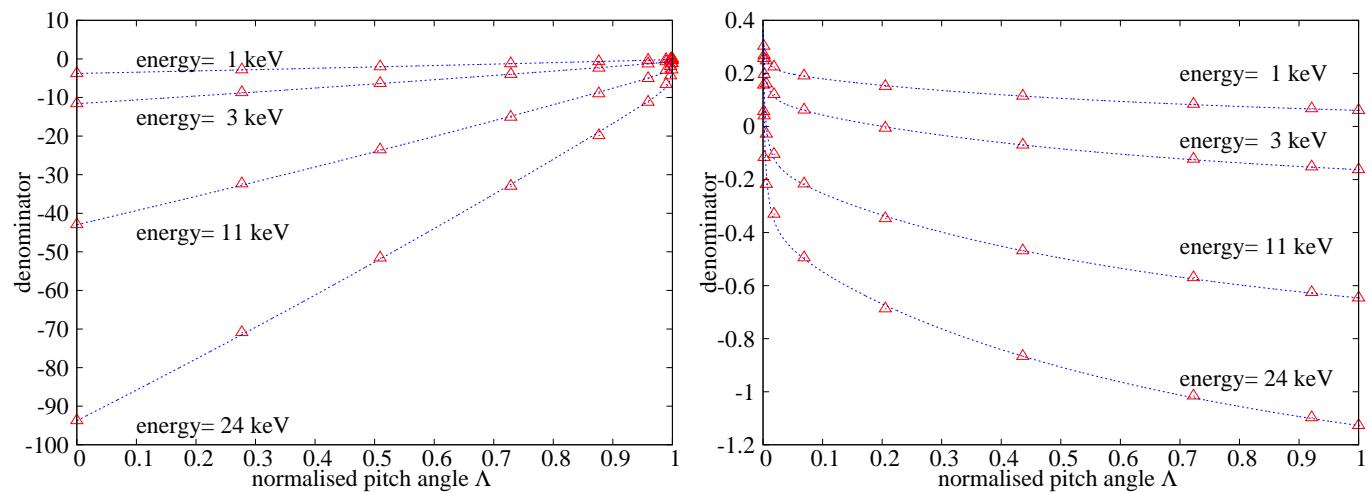

Fig. 7. Original HAGIS-data (triangles) compared to the rational interpolated denominator for passing electrons with $k=1$ (left) and trapped electrons $k=1$ (right)

As a test and benchmark case, the Landau damping problem is solved with this rational interpolation technique:

$$
2 \pi \int d v_{\perp} v_{\perp} \int d v_{\|} \frac{\partial F_{0}}{\partial E} \frac{\omega}{\omega-v_{\|} k_{\|}}=\frac{n}{T} \zeta Z(\zeta)
$$

with

$$
\zeta=\frac{\omega}{k_{\|} v_{t h} \sqrt{2}} ; \quad v_{t h}=\sqrt{T / m}
$$

and

$$
Z(\zeta)=\frac{1}{\sqrt{\pi}} \int_{-\infty}^{\infty} \frac{d u e^{-u^{2}}}{u-\zeta}=2 i e^{-\zeta^{2}} \int_{-\infty}^{i \zeta} d t e^{-t^{2}}=i \sqrt{\pi} e^{-\zeta^{2}}(1+\operatorname{Erf}(i \zeta))
$$

Figure 8 shows that the analytical result as given by formula 18 is very well recovered by the numerical integration using the rational interpolation technique described above.

Having done this rational interpolation in $\Lambda$ for each energy point, the location of the resonance is known accurately (see figure 9). This information is needed for the Cauchy principal value integration and furthermore it allows to readjust the energy grid. 

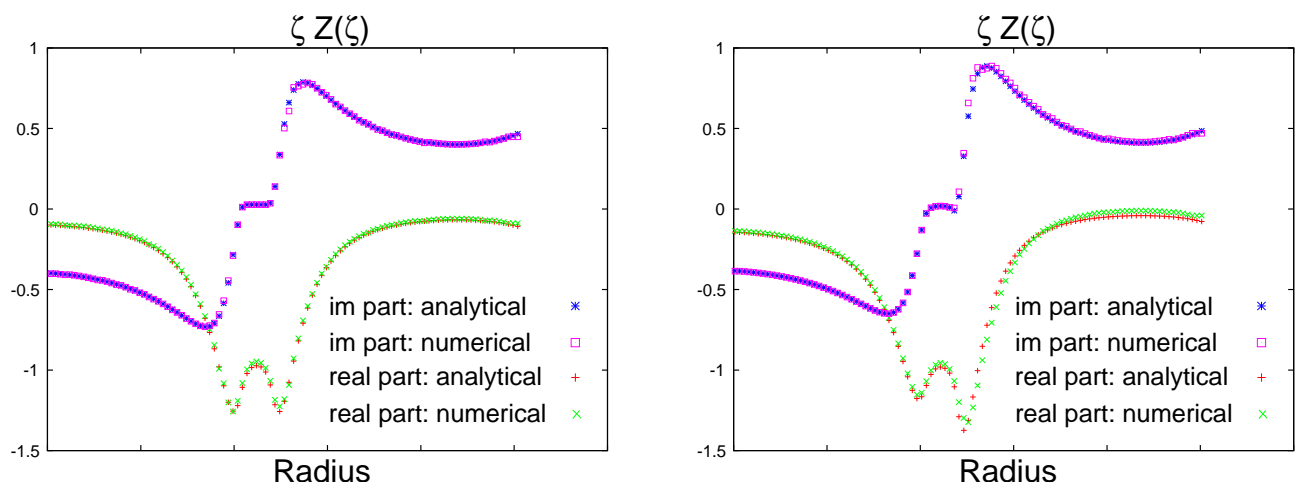

Fig. 8. Comparison of the numerical vs. analytical contribution of the velocity space integral dependent on the radial position for $\gamma / \omega=-0.1 \%$ (left) and $\gamma / \omega=-10 \%$ (right)

For a typical test equilibrium (the exact parameters are shown in the radiative damping benchmark) the lines of resonance in velocity space are shown in figure 9 for thermal electrons (left) and $\alpha$-particles (right). The frequency chosen is the TAE frequency. There are no resonances with the background ions in this case. Therefore, for the ions the lowest order solution for $h_{i}$ is sufficient and no integration over velocity space has to be performed. One can see that for circulating electrons there is a $k=0$ resonance due to the matching of $\omega$ and $\omega_{D}$ and two sideband resonances due to $k \pm 1$. There are also contributions from trapped electrons. For the $\alpha$-particles only co-passing and trapped resonances are shown. In order to determine if these resonances are stabilising or destabilising, one has to look at the combination of signs of $\partial F_{0} / \partial E$ and $\partial F_{0} / \partial r$ (contained in $\omega_{*}$ ).

It has been found that a 40 by 40 grid in velocity space $(\Lambda-E)$ is sufficient for
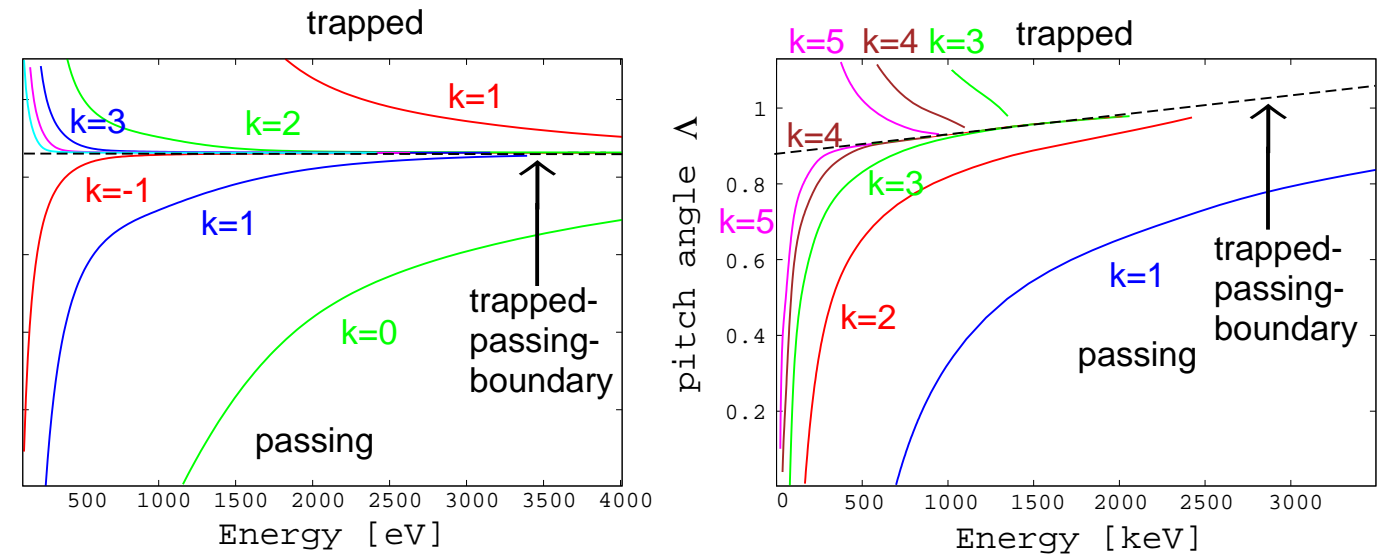

Fig. 9. Lines of resonance in the energy - pitch angle plane: electrons (left) and $\alpha$-particles, only co-passing and trapped (right).

the background species, whereas a 40 by 60 grid is needed for fast particles. All the integrations are independent of each other and therefore were parallelised. On a 16-CPU, $3.4 \mathrm{GHz}$ Linux cluster with 5 coupled poloidal harmonics, 3 
species, 10 bounce harmonics and 100 radial grid points, these integrations take about 5 minutes.

\subsection{Eigenvalue Solver}

As mentioned above, the problem is non-linear in the eigenvalue. Therefore a combination of several algorithms are applied for solving:

- As described in reference [3], the smallest eigenvalue $e_{i}(\omega)$ of the total (square) matrix $M_{i j}$ has to be identified and by using a Newton iteration, $e_{i}(\omega)=0$ has to be found. Then $\omega$ is also an eigenvalue of the whole determinant $\operatorname{Det}(\omega)$. This method works if the eigenvalue is guessed sufficiently accurate. Otherwise, the smallest $e_{i}$ can jump between different roots.

- If the eigenvalue is not known well enough, a Nyquist contour integration method $[28 ; 29 ; 30]$ is applied: a series of 8 up to 64 points on a circle around the guess are evaluated. Integrating along this contour gives the residual, that allows to determine how many roots can be found where within the contour. This algorithm seems to work relatively reliable, however the cost for typically 32-64 evaluations of the determinant is relatively high.

- As described in [2], the antenna version of the code can be employed, to find the approximated eigenvalues of the system. Usually, the antenna-result is an excellent guess and only 8-16 points are needed for full convergence with the Nyquist solver.

\section{Benchmarks and Results}

\subsection{MHD-Limit}

The benchmarks carried out in this section are all TAEs. These modes attract great interest and concern when the stability of a burning tokamak plasma is predicted. It was shown numerically and analytically how toroidal geometry breaks up the continuous Alfvén spectra, generates gaps and permits global modes within these gaps. These global modes can be driven unstable by energetic particles if the mode frequency is close to either the transit, bounce or drift frequency of the energetic particles.

For a series of circular, numerical HELENA-based equilibria [26] with $q_{0}=$ $1.05, a=0.9 \mathrm{~m}$ (minor plasma radius), $B_{0}=5 \mathrm{~T}, n(s)=n_{0}=5 \cdot 10^{19} \mathrm{~m}^{-3}$ where $R_{0}$ (i.e. the aspect ratio) varies between $3 \mathrm{~m}$ and $5 \mathrm{~m}$, the mode frequencies for the even and the odd TAE mode are compared: LIGKA in the MHD-limit and CASTOR show perfect agreement (see figure 10). The gap size scales linearly 
with $\epsilon=a / R_{0}$ as predicted theoretically [14]. Also the mode structures, shown in figure 11 for the case $R_{0}=4 \mathrm{~m}$ are very similar to each other. The difference for the $m=3$ component might be due to the fundamental difference of the models in CASTOR and LIGKA. However, KIN2DEM [3] shows the same mode ratio as LIGKA.

For the following kinetic test cases always a successful comparison with CASTOR in the MHD limit has been carried out.
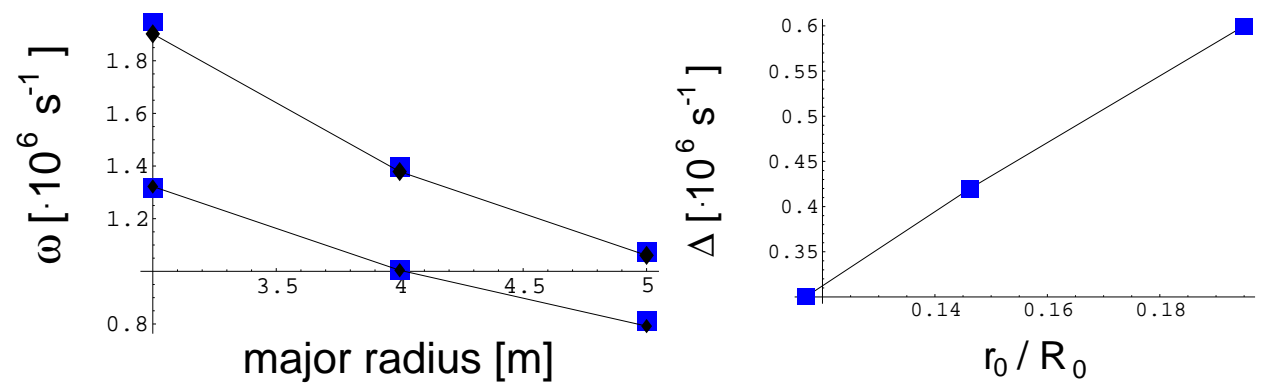

Fig. 10. Left picture: Mode frequency comparison between CASTOR (black diamonds) and LIGKA (blue squares) for the even (lower line) and odd (upper line) TAE gap mode $(m=2 / 3)$. For three equilibria with different $R_{0}$ and a fixed minor radius $(a=0.9)$ the gap size (right picture) scales linearly with $r_{0} / R_{0}$ as predicted theoretically.
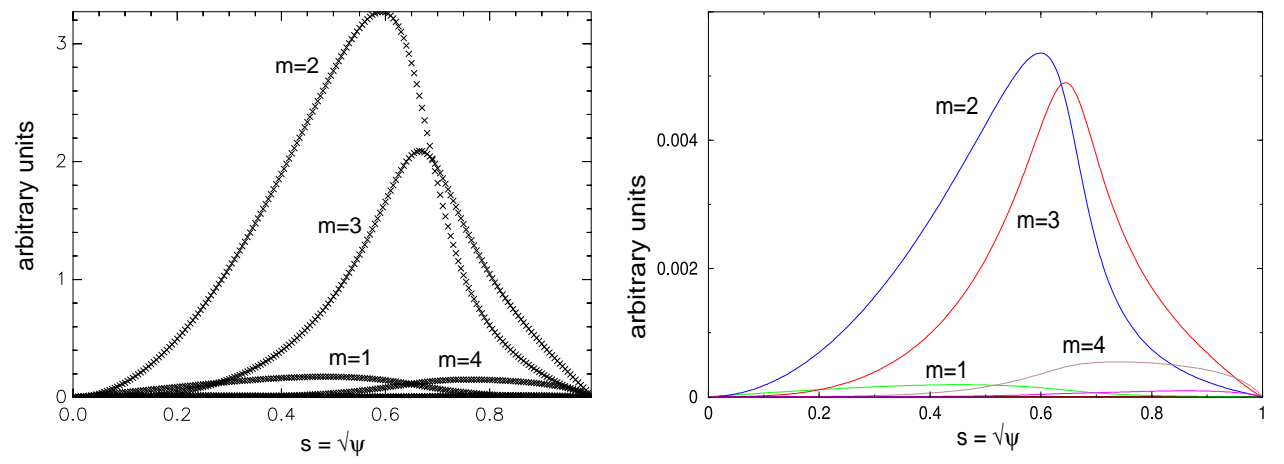

Fig. 11. Eigenfunctions of the TAE gap mode, LIGKA (left) vs. CASTOR(right)

\subsection{Hot Particle Drive}

Now kinetic modifications of the TAE mode are investigated. In order to do so a population of super-thermal Maxwellian hydrogen ions is added to the system. The parameters for this third species are chosen to be:

$$
p_{\text {hot }}=p(0) e^{-\psi / 0.09}
$$

with a constant temperature profile $T_{h}$. Following Qin's choice [3] for the temperature of the hot ions at the magnetic axis, $v_{t h / h o t}=\sqrt{2 T_{h o t / 0} / m}$ is 
varied over the interval

$$
0.5<v_{t h / h o t} / v_{A}<1.5
$$

and the poloidal $\beta_{\text {hot } / 0}=2 \mu_{0} p_{\text {hot } / 0} / B_{\theta / a}$ at the magnetic axis is chosen as 1 . The background temperature is set to:

$$
T_{i}=T_{e}=\frac{1}{2}(1-\psi)^{2} \mathrm{keV}
$$

The results for this case are shown in figure 12: the growth rate increases with the fast particle velocity and the real part of the frequency is shifted. The re-
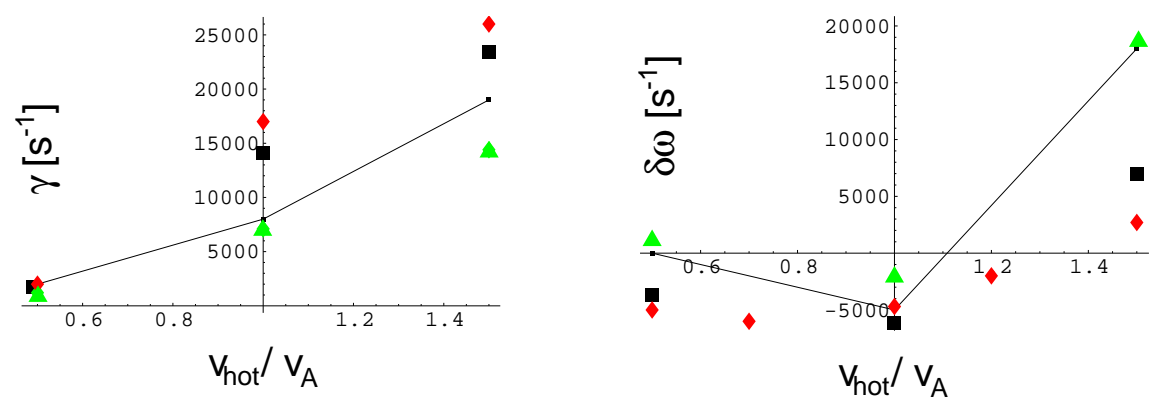

Fig. 12. Growth rate (left) and frequency shift (right) of the $(m=2,3)$ TAE mode: KIN2DEM (black line) and CAS3D-K (red diamonds) vs. LIGKA with (green triangles) and without (black squares) finite orbit width effects

sults obtained with LIGKA agree reasonably well with other codes which are capable of dealing with fast particle effects in different approximations: the red diamonds in figure 12 represent CAS3D3K results [33]. CAS3D3K is a perturbative kinetic MHD code based on CAS3D [34; 35] which is a linear, ideal, 3-dimensional MHD code, here applied in the $2 \mathrm{~d}$ tokamak geometry limit. The fast particle treatment of CAS3D3K is based on a drift kinetic description with zero banana orbit width. When the banana width is also neglected in LIGKA (black squares), an acceptable agreement of both codes is found. The remaining discrepancy can be attributed to the fact that $E_{\|}$and FLR-effects are missing in CAS3D3-K. When the finite orbit widths are switched on in LIGKA (green triangles), the growth rate decreases considerably, especially for higher $v_{t h / h o t}$. It is even smaller than predicted by KIN2DEM (solid line), which uses a Taylor expansion in the banana width. This also agrees with the fact that for large banana widths, the Taylor expansion becomes inaccurate and underestimates the stabilising influence.

\subsection{Mode Damping}

In the test case above the fast particle drive exceeded by far the damping effects. However, in order to predict stability limits, the background damping 
has to be understood. Therefore, three main damping mechanisms are now investigated: Landau damping, continuum damping and radiative damping. Collisional damping cannot be considered with the present version of LIGKA.

\subsubsection{Landau Damping}

As seen in figure 9 there are typically resonances of electrons with the TAEmode. For a Maxwellian background distribution function this means that the mode is Landau damped.

Based on the JET limiter discharge \#42979@10.12s with $B 0=3.53, R_{0}=$ $2.96 \mathrm{~m}, q 0=0.87, a=1.0 \mathrm{~m}, n_{0}=3.727 \cdot 10^{19}$ and $n=1$ with the density, temperature and q-profile given in figure 13 the damping rate of the TAE mode in the $\mathrm{m}=2,3$ gap is calculated. Figure 14 shows the benchmark
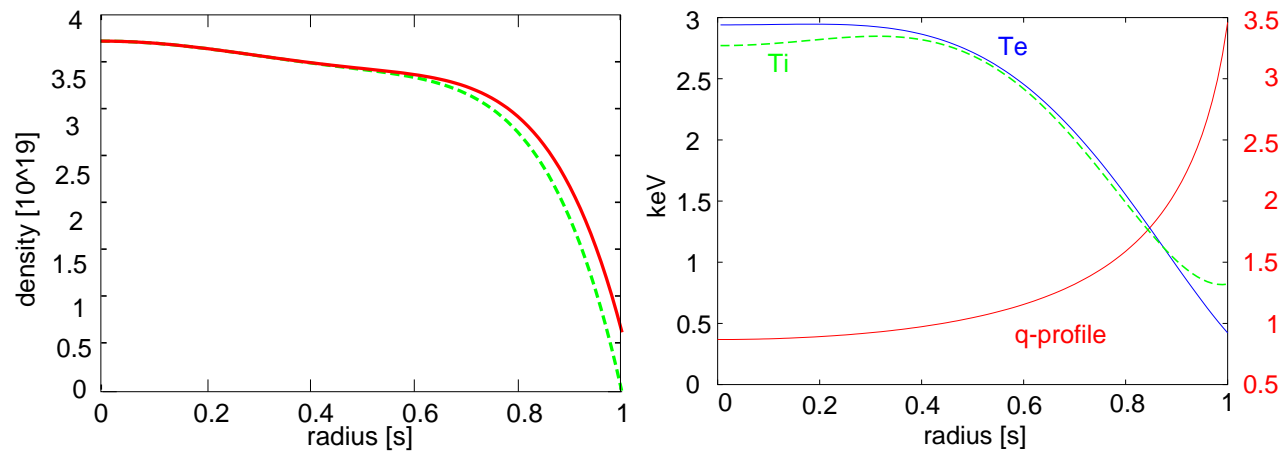

Fig. 13. Density profile for an open (solid, red) and a closed gap (dashed, green); electron and ion temperatures and the q-profile are shown on the right.

of LIGKA, CAS3D-K and analytical formulae in form of a mass isotope scan as performed in JET experiments [19]. The variation of isotope mass changes $v_{A} \sim \sqrt{1 / m_{i}}$ and also the damping rate $\gamma / \omega \sim \sqrt{\left(\beta_{e} m_{e} / m_{i}\right)}[16]$. Acceptable agreement is found. The differences however between CAS3D-K and LIGKA can be contributed to the missing of $E_{\|}$and/or background FLR effects in the drift-kinetic CAS3D-K (although the non-ideal parameter $\lambda$ which controls the radiative damping, is relatively small in this case). The modes structure is found to be an almost pure TAE mode and no mode conversion in the plasma centre was obtained. The damping rate is roughly an order of magnitude lower than the PENN prediction and the measured damping rate [19]. The scaling with $\sqrt{\left(1 / m_{i}\right)}$ however is reproduced. The reason for this difference might be the following one: PENN solves a 6-th order differential equation (fast compressional wave is kept) whereas LIGKA solves a 4-th order system (fast wave is filtered out by keeping $A_{\|}$only). In the TAE frequency range with almost pure shear Alfvén wave physics, the fast wave plays a vanishing role. However, it is known that a 6th order system allows spurious solutions which are very hard to control, especially at the magnetic axis. Thus although LIGKA captures not the same comprehensive physics as PENN it is far better suited 
to the the TAE damping problem due to its 1-d finite element discretisation and Fourier decomposition in the other two coordinates. The lack of a vacuum region in LIGKA cannot explain the crucial difference: PENN identifies mode conversion in the plasma centre as the most important damping mechanism. However, in order to further validate LIGKA with experimental data, LIGKA is about to be extended with a vacuum region.

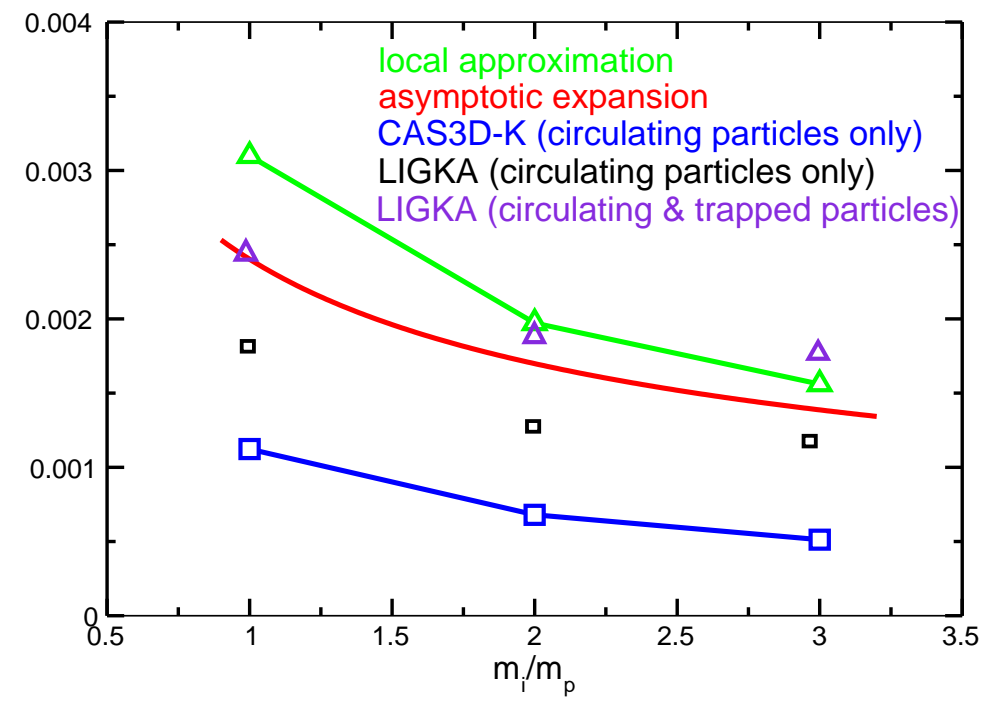

Fig. 14. Benchmark of CAS3D-K and LIGKA against analytical formulae

\subsubsection{Continuum damping at the edge}

When comparing with experimental data it has to be kept in mind that there is a relatively high uncertainty in the measured edge density profiles. Therefore it cannot be distinguished reliably if the gap is open or closed.

If the density at the edge is slightly changed within the experimental error bars (see dashed line in figure 13) the gap is closed at the edge and the mode hits the continuum. The eigenfunction is shown in figure 15. On can see a coupling to the KAW wave very close to the edge. The damping rate increases significantly to $0.7 \%$. That means that damping at the plasma edge is found to be the dominant damping mechanism, in agreement with reference [22].

The maximum value of $k_{\perp} \varrho_{i} \approx 0.28$. Thererfore the FLR expansion is justified here.

It has to be kept in mind that LIGKA is a fixed boundary code with no vacuum region. Therefore some important physics at the plasma-vacuum boundary might be missed. LIGKA has to be improved in this respect in order to determine if the discrepancy to other codes and the experiment is due this simplifiction at the edge.

Furthermore, the present version of LIGKA cannot deal with X-point configurations. Since there is a relatively strong dependence on the edge details it is expected that the damping rates change quantitatively due to a stronger 
coupling to neighbouring poloidal harmonics near the plasma edge resulting most probably in rather higher than lower damping rates. However, it will not change the qualitative result that the main damping comes from the edge region and not from mode conversion in the plasma centre.

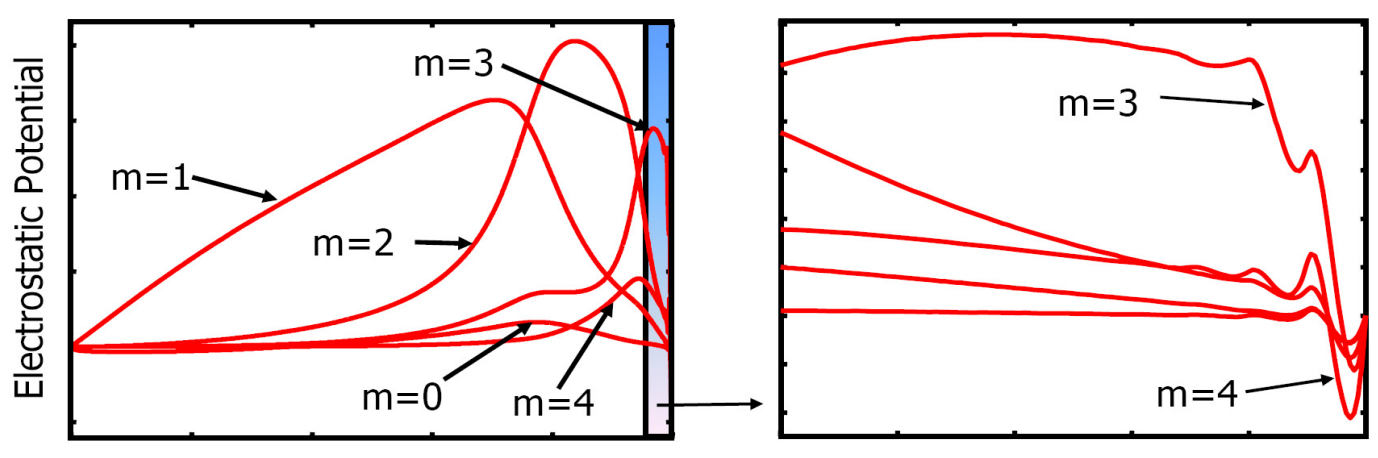

Radius

Fig. 15. Eigenfunction for the closed gap case (left) and magnified edge region (right)

\subsubsection{Radiative Damping}

The last damping mechanism investigated is radiative damping. It is controlled by the non-ideal parameter $\lambda$

$$
\lambda=\frac{4 m S \varrho_{i}}{r_{m} \hat{\epsilon}^{3 / 2}} \sqrt{\frac{3}{4}+\frac{T_{e}}{T_{i}}}
$$

with $S=r q^{\prime}(r) / q(r)$ being the magnetic shear, $\hat{\epsilon}=5 r_{m} / 2 R_{0}$ and $r_{m}$ the radial location of the gap surface. If $\lambda$ increases the coupling of the TAE mode and the KAW increases and short wavelength features of the KAW 'tunnel' into the TAE. This tunnelling takes place at the location of the gap, where the TAE is close to the continuum.

For benchmarking, an equilibrium based on the JET discharge \#38573@5.0s with the profiles described in reference [22] is chosen. By changing the ion temperature, the ion gyroradius is varied from $1 \mathrm{~mm}$ up to $5.5 \mathrm{~mm}$. The resulting damping rate is plotted in figure 16. For comparison the damping rate calculated with a simple code based on the reduced kinetic model (RKM) [22] are also shown. For this test case the equilibrium was simplified to a shiftedcircle geometry.

Very good agreement was found, despite the fact that the RKM model also includes a simple model for collisional damping which is missing in LIGKA. This is in agreement with the estimate that collisional damping gives only relatively small correction in this case. 

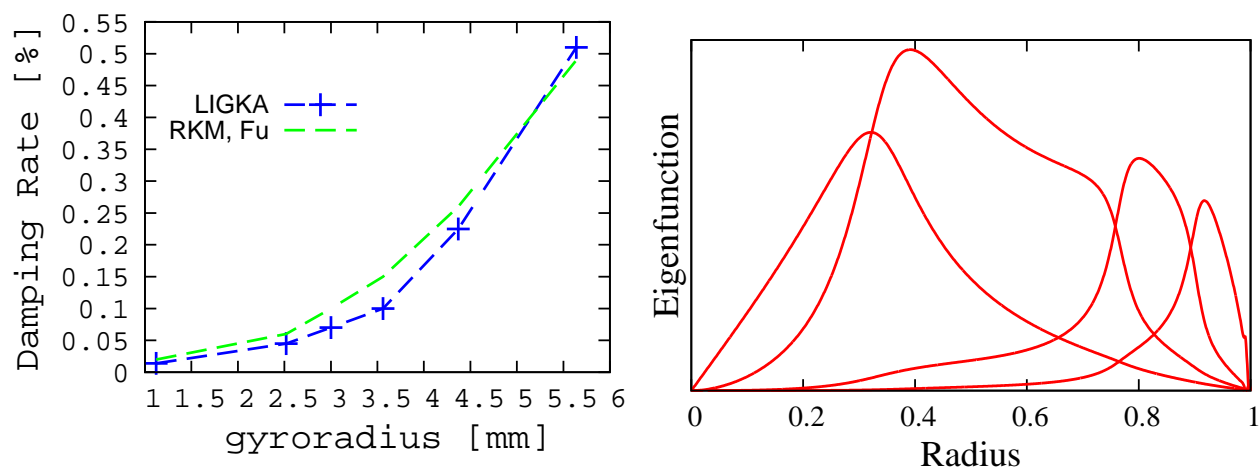

Fig. 16. Damping rate in dependence of the gyroradius for a circular equilibrium based on JET shot \#38573@5.0s (left). Eigenfunction calculated by LIGKA for $\varrho=1 \mathrm{~mm}$ (right)

Three corresponding eigenfunctions are shown in figures 16 and 17 for the gyroradii $\varrho=1 \mathrm{~mm}, 3 \mathrm{~mm}$ (experimental case) and $5.5 \mathrm{~mm}$. It can be seen how the KAW tunnels with increasing gyroradius more and more into the TAE near the gap location. A similar behaviour, however less strong, was found with the RKM code. This fact may explain the slightly stronger dependence of the damping rate on the gyroradius found by LIGKA.

For the mode in figure 17 , the maximum value of $k_{\perp} \varrho_{i} \approx 0.2$. Thus also here the FLR expansion is well justified.

In addition to the previous section, it should be mentioned that in this case the gap is open. Both codes were used to calculate a damping rate also for a closed gap situation by slightly changing the density profile at the edge. The RKM code found a considerable higher damping rate $0.5 \%,[22]$ and also LIGKA found $0.72 \%$. Again, in agreement with previous statements, damping at the edge is found to be dominant for a closed gap.
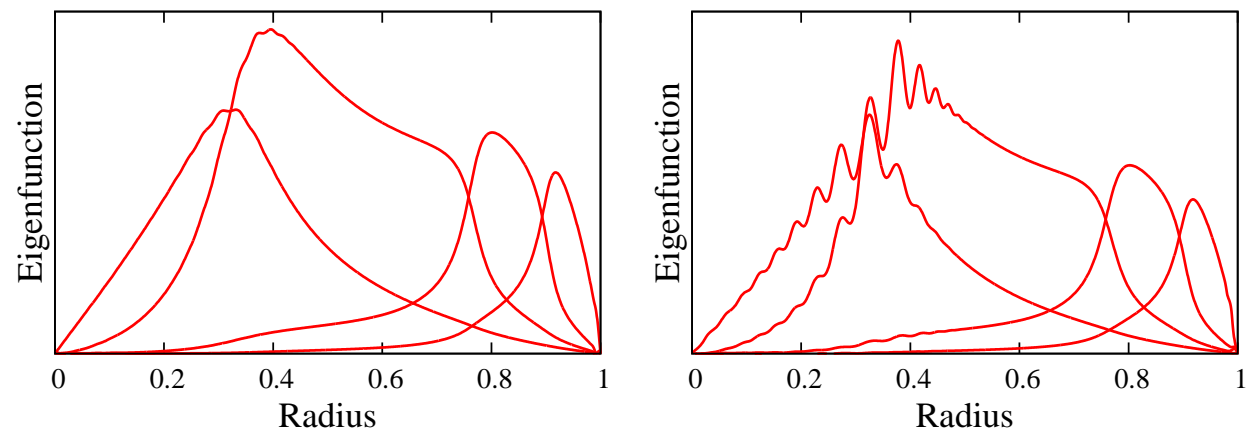

Fig. 17. Eigenfunction calculated by LIGKA for $\varrho=3 \mathrm{~mm}$ (left) and $\varrho=5.5 \mathrm{~mm}$ (right) 


\section{Conclusions}

After numerous improvements and extensions since the first version in 2003, LIGKA is now benchmarked against analytical results and other numerical codes in the most important limits and for all key-physics elements: the idealMHD limit, the reduced kinetic model, fast particle drive, Landau damping, continuum damping and radiative damping.

The benchmarks with PENN were not successful so far. This could have the following reasons: unlike PENN, LIGKA is no antenna-code with a proper vacuum region. Therefore LIGKA might miss some important phyiscs at the plasma edge. Furthermore, LIGKA looks for eigenfunctions of a certain plasma configuration whereas PENN has driven modes as solutions. It has to be investigated if these two different concepts contribute to the difference in the results. An extension of LIGKA into a proper antenna code will help to clarify this point.

However, for open gap cases where edge effects are not important, LIGKA cannot find any mode conversion in the plasma centre. This result is in agreement with analytical estimates, CASTOR-K and the 'reduced kinetic model' code and therefore contradicts the PENN results. As pointed out at the end of chapter 4.3.1, LIGKA's physics model is better adopted to shear Alfvén problems and therefore its equations are easier to solve numerically. Since also good agreement with analytical and simple kinetic models in the appropriate limits is found, LIGKA's results should be more robust and reliable for this particular problem.

As occasionally indicated before, there are numerous improvements of LIGKA on the way: the most important improvements will be an extension to the low-frequency-sound-wave regime, an extension for the vacuum region and a parallel matrix solver to allow for more poloidal harmonics as required for medium and high-n modes. 


\section{References}

[1] Lauber, Ph.,'Linear Gyrokinetic Description of Fast Particle Effects on the MHD Stability in Tokamaks', Ph.D. Thesis, TU München, (2003)

[2] Ph. Lauber, S. Günter, S.D. Pinches, Phys. Plasmas 12, 122501 (2005)

[3] H. Qin, 'Gyrokinetic Theory and Computational Methods for Electromagnetic Perturbations in Tokamaks', Ph.D. Thesis, Princeton University (1998)

[4] H. Qin, W.M. Tang, G.Rewoldt, Phys. Plasmas 5, 1035 (1998)

[5] H. Qin, W.M. Tang, G.Rewoldt, Phys. Plasmas 6, 2544 (1999)

[6] H. Qin, W.M. Tang, W.W.Lee, Phys. Plasmas 7, 4433 (2000)

[7] J.W. Connor, R.J Hastie, J.B. Taylor, Phys. Rev. Lett.40, 396 (1978)

[8] W.M. Tang, J.W. Connor, R.J. Hastie., Nucl. Fusion 20, 1439 (1980)

[9] G. Rewoldt, private communication

[10] R. Marchand, PhD Thesis, Princeton University (1979)

[11] H.L Berk, R.R. Mett, and D.M. Lindberg, Phys. Fluids B 5, 3969 (1993)

[12] , H.L. Berk, private communication

[13] C.Z. Cheng, L. Chen, M.S. Chance, Annals of Physics 161, 21 (1985)

[14] C.Z. Cheng, M.S. Chance, Phys. Fluids 29, 11 (1986)

[15] C.Z. Cheng, M.S. Chance, J. Comp. Phys 71, 124 (1987)

[16] G.Y. Fu, J.W. VanDam, Phys. Fluids 1, 1949 (1989)

[17] R.R. Mett, S.M. Mahajan, Phys. Fluids B 4, 2885 (1992)

[18] D. Borba, W. Kerner, J. Comput. Phys 153, 101 (1999)

[19] A. Fasoli, A. Jaun, D. Testa, Phys. Lett. A 265, 288 (2000)

[20] D. Borba et al., Nucl. Fusion 42, 1029 (2002)

[21] A. Jaun et al., CPC 92, 153 (1995)

[22] G.Y. Fu,H. L. Berk, A. Pletzer, Phys. Plasmas 12, 082505 (2005)

[23] S. D. Pinches, Ph.D. Thesis, The University of Nottingham, (1996)

[24] S. D. Pinches et al., CPC 111, 131 (1998)

[25] J. Candy, M.N. Rosenbluth, Phys. Plasmas 1, 356 (1994)

[26] G.T.A. Huysmans. J.P. Goedbloed, W. Kerner, Proc. CP90 Conf. on Comp Phys.Proc., Wolrd Scientific Publ.Co., p. 371 (1991)

[27] S. Brunner, J. Vaclavik, Phys. Fluids B 5, 1695 (1993)

[28] M.P. Carpentier, A.F. Dos Santos, J. Comput. Phys. 45, 210 (1982)

[29] B. Davies, J. Comput. Phys. 66, 36 (1986)

[30] S. Brunner, Private Communication (2001)

[31] P.R. Graves-Morris, T.R. Hopkins, Numer. Math. 36, 111 (1981)

[32] I.M. Longman, MTCA 12, 205 (1958)

[33] A. Koenies, IAEA TCM Meeting, Goeteborg (2001)

[34] C. Nuehrenberg, Phys. Plasmas 6, 137 (1998)

[35] C. Nuehrenberg, Plasma Phys. Control. Fusion 41, 1055 (1999) 